\title{
Observing shadow motions: Resonant activity within the observer's motor system?
}

\author{
Kaat Alaerts, Tinne Van Aggelpoel, Stephan P. Swinnen, Nicole Wenderoth* \\ Motor Control Laboratory, Research Center of Movement Control and Neuroplasticity, Department of Biomedical Kinesiology, Group Biomedical Sciences, \\ Katholieke Universtiteit Leuven, Tervuursevest 101, B-3001 Heverlee, Belgium
}

\section{A R T I C L E I N F O}

\section{Article history:}

Received 3 April 2009

Received in revised form 10 June 2009

Accepted 18 June 2009

\section{Keywords:}

Mirror system

Action observation

Cortical excitability

Transcranial magnetic stimulation

Biological motion

Shadow animation

\begin{abstract}
A B S T R A C T
Several studies have demonstrated that the human motor cortex is activated by the mere observation of actions performed by others. In the present study, we explored whether the perception of 'impoverished motion stimuli', such as shadow animations, is sufficient to activate motor areas. To do so, transcranial magnetic stimulation (TMS) was applied over the hand area of the primary motor cortex (M1) while subjects observed shadow animations depicting finger motions. Data showed that resonant motor responses in $\mathrm{M} 1$ were only found when a biological effector was recognized from the observed shadow animation. Interestingly, M1 responses were similar for observing shadow or real motions. Therefore, the loss of 'pictorial' movement features in a shadow animation appeared to have no effect on motor resonance in M1. In summary, these findings suggest that the 'recognition' of biological motion from sparse visual input is both necessary and sufficient to recruit motor areas. This supports the hypothesis that the motor system is involved in recognizing the actions performed by others.
\end{abstract}

(c) 2009 Elsevier Ireland Ltd. All rights reserved.
Mirror neurons discharge when an action is performed but also when the same action is observed [5]. Several neurophysiological and neuro-imaging experiments showed that a similar mirror mechanism also exists in the human brain $[3,6,7,10]$. Ever since its discovery, this mechanism is hypothesised to play an important role in action recognition by matching visual representations of observed actions to motor plans [13]. However, linking these phenomena, suggests that any visual input depicting biological motions should be sufficient to mediate simulating activity in the observer's motor system. In this context, a previous transcranial magnetic stimulation (TMS) study showed that the mere observation of 'implied' motion stimuli (i.e., static snapshots of hands showing a grip action) was able to induce an increase in resonant motor activity as compared with observation of resting, relaxed hands [18].

To explore this property further, the present study used TMS to measure activity modulations of primary motor cortex (M1) upon the perception of 'impoverished motion stimuli', namely shadow animations of a biological finger motion. Although shadow animations are deprived of any 'pictorial' movement information, such as overt muscle contractions or the physical appearance of a human hand, they can readily be identified as originating from a biological actor. Given that shadow animations depict actions; is their perception sufficient to recruit the observer's motor system? Or are

\footnotetext{
* Corresponding author. Tel.: +32 163291 57; fax: +32 16329197 E-mail address: Nici.Wenderoth@faber.kuleuven.be (N. Wenderoth).
}

these stimuli too simplified to drive neural activity in mirror motor mapping areas? To answer this question, resonant motor responses were assessed in the hand area of M1 while subjects observed (i) a real index finger motion (ii) an easily recognizable shadow animation of this motion, and (iii) an unrecognizable shadow animation of a finger action (see Fig. 1).

In addition, we investigated whether the attribution of a biological origin/notion to a previously unrecognizable shadow animation affects the recruitment of the observer's motor system. To do so, M1 responses were assessed a second time for the 'unrecognizable shadow condition', after subjects were familiarized with the finger motion underlying the previously unrecognizable shadow animation (i.e., by revealing the actual biological motion that it depicted). If the motor system is involved in action recognition, we expect motor resonant responses to become apparent only in the post-familiarization measurement.

Participants were 7 male and 13 female volunteers (age range 19-48, mean age 25) without any overt sensorimotor deficits. All participants were right-handed, as assessed with the Edinburgh Handedness Questionnaire [11] and were naive about the purpose of the experiment. Written informed consent was obtained before the experiment and participants were screened for potential risk of adverse effects during TMS. The study was approved by the Ethics Committee of the Katholieke Universiteit Leuven in accordance with the Declaration of Helsinki [12].

Motor-evoked potentials (MEPs) were recorded simultaneously from the first dorsal interosseus (FDI) (index finger muscle) and the extensor carpi radialis (ECR) (wrist muscle) of the right hand. 
Only the FDI, not the ECR muscle is involved in the presented 'finger abduction/adduction movements' (see below). To assess MEPs from these muscles, a surface electromyography (EMG) was recorded with $\mathrm{Ag}-\mathrm{AgCl}$ electrodes (Blue Sensor SP), placed over the middle portion of the muscle belly and aligned with the longitudinal axis of the muscle. Responses were sampled at $5000 \mathrm{~Hz}$ (CED Power 1401, Cambridge Electronic Design, UK) amplified, band-pass filtered $(30-1500 \mathrm{~Hz})$, and stored on a PC for off-line analysis. Pre-stimulus EMG recordings were used to assess the presence of unwanted background EMG activity in the $50 \mathrm{~ms}$ preceding the magnetic pulse. Focal TMS was performed by means of a $70 \mathrm{~mm}$ figure of eight coil connected to a Magstim 200 stimulator (Magstim, Whitland, Dyfed, UK). The coil was positioned over the left hemisphere, tangentially to the scalp with the handle pointing backward and laterally at $45^{\circ}$ away from the mid-sagittal line, such that the induced current flow was in a posterior-anterior direction, i.e., approximately perpendicular to the line of the central sulcus. The optimal scalp position was defined as the position from which MEPs with maximal amplitude were recorded in the right FDI. The rest motor threshold (rMT) was defined as the lowest stimulus intensity evoking MEPs in the right FDI with an amplitude of at least $50 \mu \mathrm{V}$ in 5 out of 10 consecutive stimuli [14]. For all experimental trials, the stimulation intensity was $130 \%$ of the subjects' rMT and ranged from $40 \%$ to $84 \%$ (mean $59 \%$ ) of the maximum stimulator output. Though parameter setting procedures were prioritised for FDI, MEPs were simultaneously obtained for the ECR serving as a control muscle. Signal Software
(2.02 Version, Cambridge Electronic Design, UK) was used for TMS triggering and EMG recordings.

Participants were seated in front of a Dell P992 monitor (resolution $1024 \times 768$ pixels; refresh frequency $60 \mathrm{~Hz}$ ) on which video clips were displayed with a frame rate of $25 \mathrm{~Hz}$. Video presentation timing was controlled by Blaxton Video Capture software (South Yorkshire, UK). Before the experiment, video clips were presented to the subjects to familiarize them with the experimental stimuli. Importantly however, no explicit information was provided on the content of the videos (i.e., whether or not the presented stimuli were related to finger actions). During the session, they were instructed to keep their hands and forearms as relaxed as possible and to pay full attention to the video presented. Vision of their own hand and forearm was never allowed. Muscle relaxation was monitored, and, whenever increased EMG activity became apparent during data collection, the trial was discarded and repeated.

The experimental stimuli consisted of three digital video clips representing abduction/adduction movements of the right index finger (female model) against a white background. The first video showed a real finger motion, which required the index finger to move rhythmically from $0^{\circ}$ (adducted) to approximately $40^{\circ}$ (abducted) at a frequency of $0.5 \mathrm{~Hz}$ and for a total duration of $10 \mathrm{~s}$ (real motion) (Fig. 1A). The second video showed an easily recognizable shadow animation of the finger action (recognizable shadow) (Fig. 1B) whereas in the third video, a shadow of a rotated variant of the finger action was presented, from which it was difficult

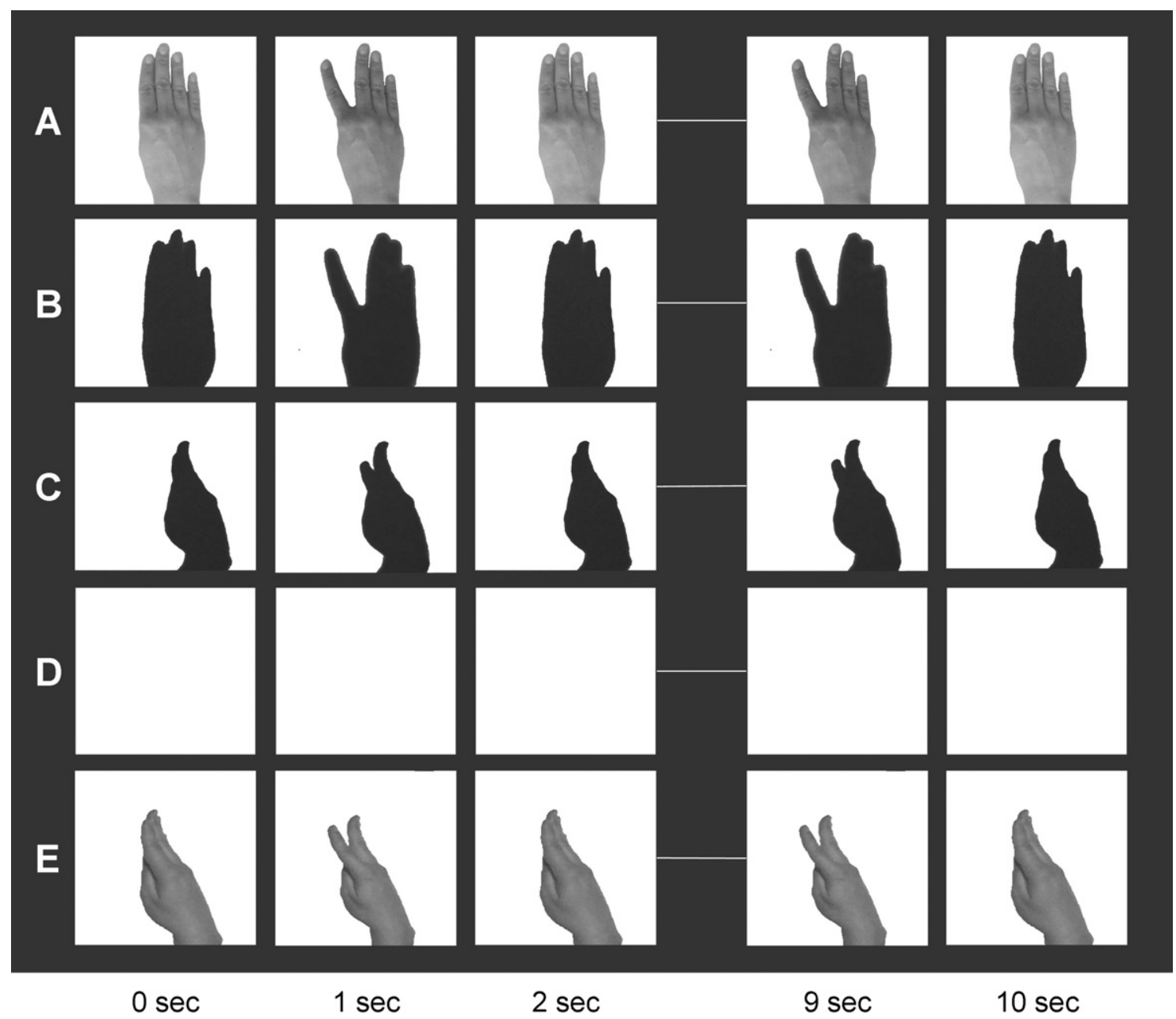

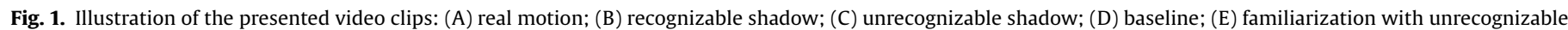
shadow. 
to recognize the actual action it depicted (unrecognizable shadow) (Fig. 1C). Debriefing of the participants revealed that subjects were indeed able to recognize the 'recognizable shadow' as originating from an actual index finger motion, whereas the 'unrecognizable shadow', although assumed to be biological by some of the subjects, was never recognized to originate from a motion of the index finger. Finally, also a control video of the same duration was made which showed only a white background (baseline) (Fig. 1D). Each of the four video clips was presented 10 times in blocks of four, with the block presentation order randomized within and across subjects. During the observation of each video clip, two single TMS pulses were delivered at random time points (with an inter stimulus interval of $5 \mathrm{~s}$ ), such that stimulation was applied when the index finger in the video reached peak abduction. For each subject and each of the 4 conditions, 20 MEPs were recorded, resulting in a total of 80 MEPs.

Nineteen (of the 20) subjects participated in part 2 of the experiment (one female subject ceased the experimental session early). Subjects were familiarized with the initially unrecognizable shadow animation by showing the actual finger motion that it depicted in a block of 10 videos (duration of each video: $10 \mathrm{~s}$ ) (Fig. 1E). Following this 'familiarization phase' (total duration: less than $2 \mathrm{~min}$ ), the initially unrecognizable shadow animation was presented again to assess the effect of familiarization. The video was presented 10 times, with two TMS pulses delivered within each trial.

The total experimental session (part $1+$ part 2) lasted approximately $60 \mathrm{~min}$ with a small pause after $30 \mathrm{~min}$.

From the EMG data, peak-to-peak MEP amplitudes were determined. Additionally, background EMG was quantified by calculating the root-mean-square error (RMSE) for the $50 \mathrm{~ms}$ interval prior to TMS stimulation to ensure that subjects were completely relaxed during the stimulation. Trials were removed from the analysis when EMG RMSE scores were larger than Q3+1.5 (Q3-Q1) with Q1, Q3 being the first and third quartile considering all trials of one observation condition and subject. For FDI and ECR muscles, MEP amplitudes were averaged separately for each of the observation conditions. Since MEP size usually exhibits large inter-individual variability, MEP amplitudes were normalized for each muscle relative to the mean baseline measure $\left(\mathrm{MEP} / \mathrm{MEP}_{\text {baseline }} \times 100\right)$ in order to make them comparable across subjects. As such, for all observation conditions, mean MEP amplitude scores (recorded from the FDI and ECR muscles) are reported (in all figures) as a percentage change from the mean MEP response recorded during the baseline observation condition. RMSE scores of the background EMG were normalized accordingly (RMSE/RMSE $E_{\text {baseline }} \times 100$ ).

Normalized peak-to-peak MEP amplitudes recorded from the different experimental conditions in part 1 were subjected to an analysis of variance (ANOVA) for repeated measurements with the factors 'Muscle' (FDI, ECR) and 'Video' (real motion, recognizable shadow, unrecognizable shadow). The sphericity assumption was confirmed using Mauchly's sphericity test for both the three-level factor 'Video' $[W(2)=.912 ; p=.445]$ and the interaction 'Muscle $\times$ Video' $[W(2)=.922 ; p=.484]$. A pre-planned comparison was performed to directly compare the evoked MEP responses from the 'real motion' and 'recognizable shadow' condition.

One sample $t$-tests were conducted to determine whether the mean MEP responses of the FDI and ECR muscle differed significantly between the baseline condition and the different observation conditions (no difference corresponds to a value not significantly different from 0$)$.

In part 2, a pre-planned comparison is performed to compare MEP responses of the FDI for the 'unrecognizable shadow' condition pre- and post-familiarization. The level of significance was set to $\alpha=0.05$. The statistical program 'Statistica 8.0' was used for all analyses (Stat. soft. inc. Tulsa, USA).

To address whether peak-to-peak MEP amplitude scores were confounded by modulations in background muscle activity, all analyses performed on the MEP amplitudes were also conducted for the corresponding normalized RMSE-scores.

MEP responses evoked from the FDI were significantly increased compared to baseline for observing the 'real motion' $[t=2.106$; $p<.05$ ] and the 'recognizable shadow' conditions [ $t=2.257 ; p<.05]$, but not for observing the 'unrecognizable shadow' condition
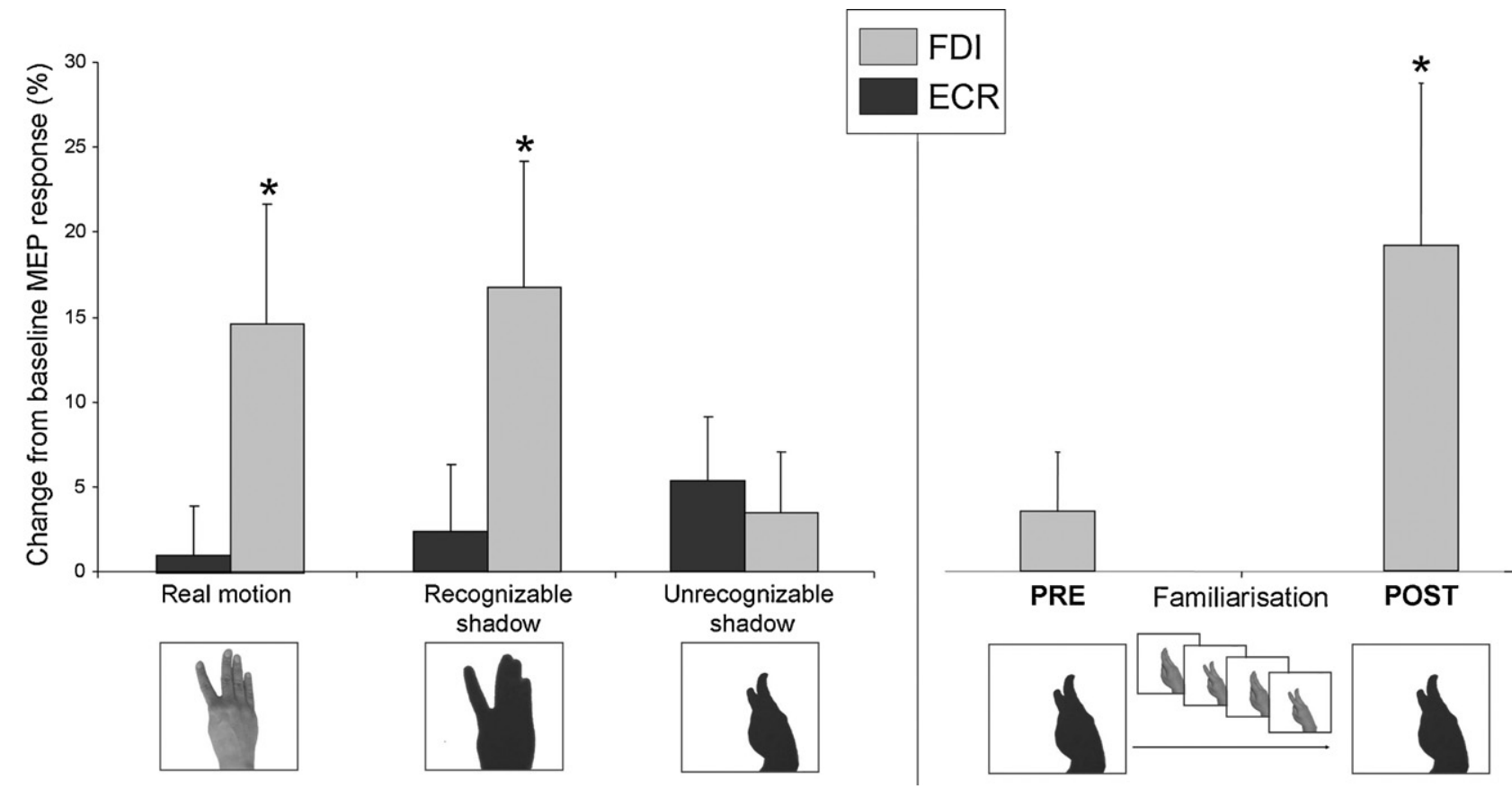

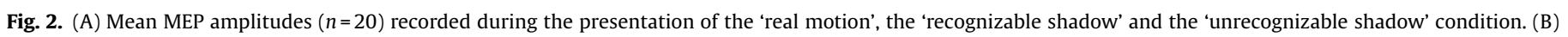

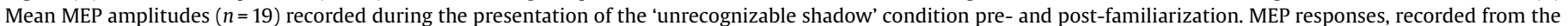

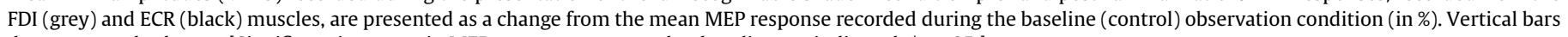
denote \pm standard error. [Significant increases in MEP response compared to baseline are indicated; ${ }^{*} p<.05$.] 
$[t=1.00 ; p=.329]$ (Fig. 2A). MEP responses evoked from the ECR muscle were comparable to baseline for all observation conditions [all, $t<1.435 ; p>$.17].

Accordingly, the significant 'Muscle $\times$ Video' interaction $[F(2,38)=3.6 ; p<.05]$ indicated further that MEP responses evoked from the FDI muscle were selectively modulated by observing the different video clips, whereas those evoked from the ECR remained relatively low and constant (Fig. $2 \mathrm{~A})$. Neither the 'Muscle' $[F(1$, $19)=2.55 ; p=.13]$ nor the 'Video' main effect $[F(2,38)=.857 ; p=.43]$ reached significance.

Observation of the 'real motion' or the 'recognizable shadow' condition evoked comparably high MEP responses from the FDI muscle. Accordingly, even a very liberal planned comparison between these two observation conditions revealed no significant difference $[F(1,19)=.137 ; p=.72]$ (Fig. 2A).

In part 2 of the experiment, 19 (of the 20) subjects were familiarized with the previously 'unrecognizable shadow' condition, by showing them the actual (finger) motion that was linked to it (Fig. 1E). After repeatedly seeing the actual motion, subjects observed the 'unrecognizable shadow' condition again to obtain the 'post-familiarization' MEP responses.

Results indicated that MEP responses, recorded prefamiliarization, were relatively low and comparable to baseline $[t=1.1 ; p=0.28]$, whereas, post-familiarization, MEP responses were shown to be significantly increased compared to baseline [t=2.1; $p=0.05$ ] (Fig. 2B). However, direct comparison between the pre- and post-familiarization conditions revealed only a trend toward a significant difference $[F=3.18 ; p=.091]$.

The background EMG was generally small and condition induced modulations were minimal, such that statistics on the RMSE scores did not reveal significant effects [for all comparisons, $p>.12$ ]. This indicates that the MEP peak-to-peak amplitude scores were not confounded by modulations in background EMG.

The present study aimed to explore whether an abstract representation of biological motion is sufficient to mediate resonant activity within the motor system of observers. To do so, TMS was used to measure activity modulations within the hand area of the primary motor cortex (M1) while subjects observed shadow animations of biological motion. Data revealed three main findings: (i) perception of shadow animations depicting a biological action appeared to be sufficient to elicit resonant activity within the observer's M1; (ii) the extent of resonant motor activity was similarly high for observing shadow or real movements; and (iii) recognition of the biological effector constituting the shadow motion was a prerequisite for mirror motor mapping to occur. As such, the 'recognition' of biological motion from sparse visual input appears to be both necessary and sufficient for mirror motor mapping to take place.

In general, our results accord to a number of TMS studies on action observation by showing that the observer's M1 is activated by the mere observation of other's actions in a highly muscle specific way, i.e., motor resonant responses were measured only in the representation area of the FDI finger muscle (involved in the finger motion), but not in the ECR wrist muscle (not recruited in the observed motion) $[1,2,6,16]$. However, expanding the results from previous studies on the properties of the human mirror system, the present study shows that even impoverished motion stimuli, such as shadow animations of biological motion can yield resonant motor responses in the observer's M1. Indirectly, these results relate to findings from a previous fMRI [15] and electro-encephalography study [17], showing increased activations in motor areas from the perception of point-light displays. Together with these data, the present findings strongly suggest that motor areas are recruited to retrieve biological motion information from sparse visual input, which in turn may contribute to the recognition of perceived actions.
In contrast to these previous studies on point-light displays, the present study directly compared the extent of resonant motor activity for observing shadow versus real motion. Interestingly, it was found that resonant motor responses were equally high for the two conditions. Therefore, the loss of pictorial motion features, such as actual muscle contractions or the physical appearance of a human hand, appeared to have no significant effect on the overall motor resonant responses in M1. This finding further underlines the assumption that the perception of 'impoverished' biological motion is sufficient to retrieve a full-blown representation of the perceived action. In this context, these data may provide an indirect explanation for the behavioural finding that the learning of complex motions equally benefits from the observation of point-light displays, compared to real motion observation [9].

As a control condition, we included the observation of a shadow animation from which no biological action could be recognized. Interestingly, motor resonant responses were generally small and comparable to baseline for this observation condition, such that the recognition of the biological effector - constituting the shadow motion - appeared to be essential for evoking resonant motor activity. Alternatively, one might argue that the lack of motor resonance for observing the unrecognizable shadow animation may reflect the smaller amount of motion that was displayed in this animation, compared to the recognizable shadow animation. However, this interpretation seems rather unlikely considering the obtained results from part 2 of the experiment: Here it was shown that when the subjects were familiarized with the formerly unrecognized shadow animation (i.e., by revealing the actual index motion that it depicted) and the same shadow animation was observed post-familiarization, motor resonant responses suddenly became apparent. As such, since the abstract visual input (and consequently the degree of displayed motion) was identical pre- and post-familiarization, the recognition of the visual input, as originating from a biological effector, appeared to be the triggering factor for excitability increases in M1 to take place. Even though our results must be interpreted with some reservations, they tentatively suggest that the mirror system is adaptive and associative to the personal visual experience of the observer. In this view, our data may extend some previous studies showing that the motor experience of an observer plays an important role in the magnitude of mirror motor responses during action observation $[4,8]$.

The present study showed that the observer's motor system 'resonates' to the observation of shadow animations depicting biological motion. Importantly, recognition of the biological effector - constituting the shadow motion - was essential for resonant motor activity to take place. However, whenever motor resonant responses were present, observation of shadow motions activated the observer's motor system to an equal extent compared to observing real motion. Overall, these data provide further evidence that action recognition is necessarily related to resonant activity within motor areas.

\section{Acknowledgements}

Support for this study was provided through grants from the Flanders Fund for Scientific Research (Projects G.0292.05, G.0577.06 and G.0749.09). This work was also supported by Grant P6/29 from the Interuniversity Attraction Poles program of the Belgian federal government.

\section{References}

[1] K. Alaerts, E. Heremans, S.P. Swinnen, N. Wenderoth, How are observed actions mapped to the observer's motor system? Influence of posture and perspective, Neuropsychologia 47 (2009) 415-422.

[2] K. Alaerts, S.P. Swinnen, N. Wenderoth, Is the human primary motor cortex activated by muscular or direction-dependent features of observed movements? Cortex, in press. 
[3] G. Buccino, F. Binkofski, G.R. Fink, L. Fadiga, L. Fogassi, V. Gallese, R.J. Seitz K. Zilles, G. Rizzolatti, H.J. Freund, Action observation activates premotor and parietal areas in a somatotopic manner: an fMRI study, European Journal of Neuroscience 13 (2001) 400-404.

[4] B. Calvo-Merino, D.E. Glaser, J. Grezes, R.E. Passingham, P. Haggard, Action observation and acquired motor skills: an fMRI study with expert dancers, Cerebral Cortex 15 (2005) 1243-1249.

[5] G. Di Pellegrino, L. Fadiga, L. Fogassi, V. Gallese, G. Rizzolatti, Understanding motor events: a neurophysiological study, Experimental Brain Research 91 (1992) 176-180.

[6] L. Fadiga, L. Fogassi, G. Pavesi, G. Rizzolatti, Motor facilitation during action observation: a magnetic stimulation study, Journal of Neurophysiology 73 (1995) 2608-2611.

[7] J. Grezes, J.L. Armony, J. Rowe, R.E. Passingham, Activations related to "mirror" and "canonical" neurones in the human brain: an fMRI study, Neuroimage 18 (2003) 928-937.

[8] B. Haslinger, P. Erhard, E. Altenmuller, U. Schroeder, H. Boecker, A.O. CeballosBaumann, Transmodal sensorimotor networks during action observation in professional pianists, Journal of Cognitive Neuroscience 17 (2005) 282-293.

[9] N.J. Hodges, S.J. Hayes, G. Breslin, A.M. Williams, An evaluation of the minimal constraining information during observation for movement reproduction, Acta Psychologica (Amst) 119 (2005) 264-282.

[10] M. Iacoboni, R.P. Woods, M. Brass, H. Bekkering, J.C. Mazziotta, G. Rizzolatti, Cortical mechanisms of human imitation, Science 286 (1999) 2526-2528.
[11] R.C. Oldfield, The assessment and analysis of handedness: the Edinburgh inventory, Neuropsychologia 9 (1971) 97-113.

[12] P.P. Rickham, Human experimentation. Code of Ethics of the World Medical Association. Declaration of Helsinki, British Medical Journal 2 (1964) 177

[13] G. Rizzolatti, L. Fadiga, V. Gallese, L. Fogassi, Premotor cortex and the recognition of motor actions, Cognitive Brain Research 3 (1996) 131-141.

[14] P.M. Rossini, A.T. Barker, A. Berardelli, M.D. Caramia, G. Caruso, R.Q. Cracco, M.R. Dimitrijevic, M. Hallett, Y. Katayama, C.H. Lucking, A.L.M. Denoordhout, C.D. Marsden, N.M.F. Murray, J.C. Rothwell, M. Swash, C. Tomberg, Noninvasive electrical and magnetic stimulation of the brain, spinal-cord and roots - basic principles and procedures for routine clinical-application - report of an IFCN Committee, Electroencephalography and Clinical Neurophysiology 91 (1994) 79-92

[15] A.P. Saygin, S.M. Wilson, D.J. Hagler Jr., E. Bates, M.I. Sereno, Point-light biological motion perception activates human premotor cortex, Journal of Neuroscience 24 (2004) 6181-6188.

[16] A.P. Strafella, T. Paus, Modulation of cortical excitability during action observation: a transcranial magnetic stimulation study, Neuroreport 11 (2000) 2289-2292.

[17] E.R. Ulloa, J.A. Pineda, Recognition of point-light biological motion: mu rhythms and mirror neuron activity, Behavioural Brain Research 183 (2007) 188-194.

[18] C. Urgesi, V. Moro, M. Candidi, S.M. Aglioti, Mapping implied body actions in the human motor system, Journal of Neuroscience 26 (2006) 7942-7949. 